\title{
Uso de drogas inhibidoras de P2Y12, clopidogrel, prasugrel y ticagrelor, en pacientes sometidos a intervenciones coronarias percutáneas en el mundo real en la Argentina. Resultados de los Registros ERACI IV y WALTZ
}

\author{
Use of P2Y12 inhibitors, clopidogrel, prasugrel and ticagrelor, in patients \\ undergoing percutaneous coronary interventions in the real world in \\ Argentina. Results from ERACI IV and WALTZ registries
}

Rodríguez-Granillo $A M^{1}$, Haiek $C^{2}$, Larribau $\mathrm{M}^{3}$, Fernández-Pereira $\mathrm{C}^{4}$, Mieres $\mathrm{J}^{5}$, Sarmiento $\mathrm{R}^{6}$, Pocoví $\mathrm{A}^{7}$, Menéndez $\mathrm{M}^{8}$, Lloberas $\mathrm{J}^{9}$, Sisu $\mathrm{E}^{10}$, Iravedra $\mathrm{J}^{11}$, Montoya $\mathrm{M}^{12}$, Rifourcat $\mathrm{I}^{13}$, Santaera $\mathrm{O}^{6},{ }^{14}$, Ming $\mathrm{Z}^{15}$, Pan $\mathrm{W}^{15}$, Rodríguez $\mathrm{AE}^{1}{ }^{16}$

\section{RESUMEN}

Objetivo. Conocer las tendencias actuales con respecto al uso de drogas antiplaquetarias (DAPT) en pacientes (ptes) con enfermedad coronaria tratados con angioplastia coronaria en la Argentina.

Métodos. Se evaluó el uso de doble antiagregación plaquetaria en 426 ptes consecutivos con enfermedad coronaria revascularizados con ATC en 15 centros de la Argentina desde 2013 a 2016, incluidos en dos registros (ERACI IV y WALTZ). Los stents implantados fueron de cromo cobalto liberador de rapamicina de segunda generación (Firebird $2^{\circledast}$ ) y de cromo cobalto $\left(\mathrm{Waltz}^{\circledR}\right.$ ), respectivamente. La indicación de revascularización de 203 arterias coronarias epicárdicas y/o enfermedad de tronco coronario no protegido (ETCI) fue criterio de inclusión para el registro ERACI IV, no así para el WALTZ, un registro de "mundo real", incluyendo síndrome coronario agudo (SCA) con elevación del STT. Los criterios de exclusión fueron mala función ventricular $<35 \%$, ATC previa con implante de stent liberador de fármacos (DES), lesiones tratadas con diámetro $<2,5 \mathrm{~mm} 0$ contraindicación para DAPT. El objetivo primario fue la incidencia de MACCE (compuesto por muerte, infarto de miocardio con y sin elevación del segmento ST-T y accidente cerebrovascular) a un año, comparando las DAPT disponibles (Clopidogrel, ticagrelor y prasugrel). Los componentes de MACCE individuales fueron objetivos secundarios, así como la incidencia de trombosis del stent y sangrado mayor. Se tomó el criterio de intención de tratar para comparar resultados de acuerdo a la tienopiridina seleccionada. Ambos registros fueron presentados a ANMAT, aprobados por las autoridades de cada centro, monitorizados por investigadores independientes y un comité de adjudicación evalúo todos los eventos. La DAPT fue obligatoria durante un año en el grupo DES y por lo menos durante 6 meses para el grupo stent desnudos (BMS). Se utilizó chi cuadrado para las variables categóricas y ANOVA para las continuas. Se discriminó de acuerdo a la presentación clínica, tipo de seguridad social (privada o pública) y tipo de stent implantado (DES o BMS) para evitar confundidores. Se utilizó el paquete estadístico SPSS 11.7. Resultados. Los ptes fueron seguidos por un año, sin pérdidas al seguimiento. 83,5\% fueron hombres, 26,7\% diabéticos y $63,4 \%$ tuvieron un SCA al ingreso (16\% SCACEST). El score de SYNTAX fue del $17,25 \pm 12,7$ y el de ERACI $12,9 \pm 11,5$. El $52,8 \%$ de los pacientes recibieron un DES. $56,4 \%$ recibió clopidogrel, $18,8 \%$ prasugrel y $24,8 \%$ ticagrelor. El grupo prasugrel tuvo un SYNTAX más elevado $(22,4 \pm 12,1)$ comparado con clopidogrel $(16,7 \pm 13,1$ y/o ticagrelor $14,5 \pm 11,1 ; p<0.001)$. A un año, no hubo diferencias en cuanto a MACCE $(p=0,32)$ ni diferencias en sangrado o stent trombosis, con una sobrevida libre de eventos mayor en el grupo prasugrel $(p=0,04)$ al compararse con ticagrelor. Ni el tipo de seguro social ni la elección del stent (BMS o DES) fueron predictores de elección de tienopiridina, aunque el ticagrelor fue, porcentualmente, la más elegida en el grupo de pacientes con SCACEST ( $p=0,04)$.

Conclusiones. En Argentina los patrones de uso de DAPT no siguen las recomendaciones actuales de las guías de tratamiento. A pesar de la evidencia contemporánea, se utiliza clopidogrel como primera elección de tienopiridinas, sin importar el cuadro clínico de ingreso, el stent implantado o el tipo de seguro social de los pacientes.

Palabras claves: DAPT, prasugrel, ticagrelor, clopidogrel, SCA, Argentina.

\section{ABSTRACT}

Objective. To know the current practice at the time of tienopiridines election in patients with coronary artery disease (CAD) undergoing percutaneous coronary interventions ( $\mathrm{PCl}$ ) in Argentina.

Methods. We evaluated the use of double antiplatelet therapy (DAPT) in 426 consecutive patients with CAD udergoing PCI in 15 sites from Argentina during 2013 to 2016, included in 2 prospective registries (ERACI IV and WALTZ). The stents implanted were of a cromo cobalt alloy (WALTZC) and 2nd generation rapamycin eluting cromo cobalt stents (ERACI IV(C). The indication for revascularization of 2 or 3 epicardial vessels or left main disease was an inclusion criteria for the ERACI IV registry but not for WALTZ, a "real world" registry, including acute coronary síndromes with ST elevation (STEMI). Exclusion criteria were ventricular ejection fraction $\leq 35 \%$, previous $\mathrm{PCI}$ with drug eluting stent (DES), treated lesions with reference diameter $\leq 2.5$ $\mathrm{mm}$ and contraindication for DAPT. The primary objective was the incidence of MACCE (the composite of death, acute myocardial infarction and stroke) at one year comparing the three available DAPT drugs (clopidogrel, prasugrel and ticagrelor). The individual components of MACCE were also evaluated as well as stent thrombosis and major bleeding. The endpoint analyses was done by the intention to treat principle. Both registries were presented to the National Health Administration of Argentin (ANMAT) and approved by the authorities of each site. A blind committee adjudicated the events and each site was monitorized by independent CRO. DAPT was mandatory during one year for DES group and up to six months for the bare metal stent (BMS) group. Chi square was used for categorical variables and ANOVA for continuous ones. We discriminate between clinical presentation, kind of social security (public or private) and type of implanted stent (DES or BMS) to avoid cofounders. SPSS 11.7 was the statistical software used for analysis.

Results. Patients were followed for one year, without loses (100\% of follow-up). $83.5 \%$ were men, $26.7 \%$ diabetics and $63.4 \%$ had a acute coronary síndrome at baseline (16\% STEMI). SYNTAX score was $17.25+12.7$ and ERACI score $12.9+11.5 .52 .8 \%$ of patients received clopidogrel, $18.8 \%$ prasugrel and $24.8 \%$ ticagrelor. Prasugrel group had a higher SYNTAX (22.4 \pm 12.1$)$ compared either with clopidogrel (16.7 \pm 13.1 $p<0.001)$ and ticagrelor $(14.5 \pm 11.1 ; p<0.001)$. At one year, there were neither differences in MACCE ( $p=0.32$ ), major bleeding or stent thrombosis, with a significantly lower incidence of any cause of death in the prasugrel group compared to ticagrelor $(p=0.04)$. Neither the type of social security nor stent type was predictor of tienopiridine selection, although ticagrelor was the drug of choice in STEMI $(p=0,04)$.

Conclusion. In Argentina the tienopiridine's use patterns do not follow current guidelines. In contrast with evidence, clopidogrel is the first choice and neither the baseline diagnosis, stent choice or social security appears to influence the tienopiridine selection.

Keywords: DAPT, prasugrel, ticagrelor, clopidogrel, acute coronary syndromes.
1. Sanatorio Otamendi, Argentina

2. Sanatorio Trinidad Quilmes, Argentina

3. Hospital Español y Policlínico de Cuyo, Mendoza, Argentina

4. Clínica IMA, Argentina

5. Sanatorio Las Lomas, Argentina

6. Hospital El Cruce, Argentina

7. Centro Médico Talar, Argentina
8. Sanatorio Plaza, Rosario, Argentina

9. Sanatorio San Miguel, San Miguel, Argentina

10. Clínica Ipensa, La Plata, Argentina

11. Clínica 25 de Mayo, Buenos Aires, Argentina

12. Clínica Sagrada Familia, Buenos Aires, Argentina

13. Instituto de Diagnóstico y Tratamiento de Enfermedades Cardiovasculares, La Plata, Argentina

14 Clínica Provincial de Merlo, Buenos Aires, Argentina
15. Microport Corp, China

16. Centro de Estudios en Cardiología Intervencionista, $\mathrm{CECl}$, Argentina

$\triangle$ Correspondencia: Dr. Rodríguez-Granillo AM. mrodriguezgranillo@centroceci.com.ar Los autores declaran no tener conflictos de intereses Recibido: 30/08/2019|Aceptado: 21/09/2019 

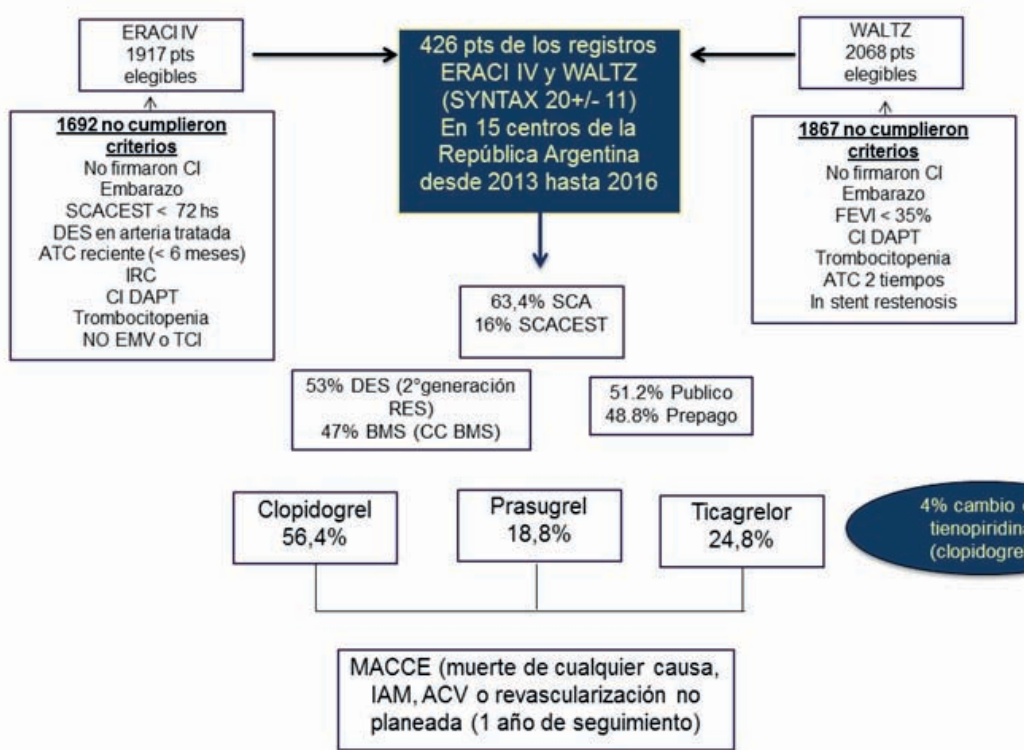

Figura 1. Diseño del registro l: consentimiento informado. SCACEST: síndrome coronario agudo con elevación del ST-T. DES: stent liberador de fármacos. ATC: angioplastia coronaria. IRC: insuficiencia renal crónica. CI DAPT: contraindicación doble antiagregación plaquetaria. EMV: enfermedad de múltiples vasos. TCI: tronco coronario izquierdo. SCA: síndrome coronario agudo. FEVI: fracción de eyección del ventrículo izquierdo. RES: stent liberador de rapamicina; CC BMS. stent convencional de cromocobalto. MACCE: eventos adversos cardiovasculares mayores. IAM: infarto agudo de miocardio; ACV: accidente cerebrovascular.

\section{INTRODUCCIÓN}

La elección entre el clopidogrel y las nuevas drogas inhibidoras de P2Y12 (prasugrel y ticagrelor) como parte del tratamiento de doble antiagregación plaquetaria (DAPT) en pacientes con cardiopatía isquémica a los que se les realiza una intervención coronaria percutánea (ICP) como método de revascularización se ha modificado en los últimos años debido a la superioridad evidenciada de estas últimas sobre el clopidogrel, a las nuevas estrategias propuestas en cuanto al stent utilizado -nuevas generaciones de stents liberadores de fármacos (DES) - y duración del tratamiento con DAPT ${ }^{1-7}$. Más allá de las conocidas indicaciones on-label a la hora de elegir uno u otro fármaco, el impacto de nueva evidencia permite imaginar nuevos rumbos terapéuticos ${ }^{8}$. Sin embargo, la evidencia en nuestro país acerca del uso y duración de la DAPT es escasa. Conocer datos del "mundo real" acerca de la elección de tienopiridinas en Argentina permite modelar el perfil de los pacientes que pueden beneficiarse con las diferentes estrategias, sobre todo en aquellos con síndromes coronarios agudos (infarto de miocardio con y sin supradesnivel del segmento ST-T) y/o score de SYNTAX elevado. El objetivo de este manuscrito es reconocer las tendencias actuales con respecto al uso de drogas inhibidoras de P2Y12 en la Argentina y el impacto de las recomendaciones de las guías de tratamiento en la práctica diaria.

\section{MATERIAL Y MÉTODOS}

\section{Diseño del estudio}

Se realizó un subanálisis de todos los pacientes incluidos en los registros ERACI IV y WALTZ, siendo ambos estudios de cohorte, prospectivos, observacionales, del "mundo real", multicéntricos y nacionales, que enrolaron 426 pacientes con enfermedad coronaria sometidos a una ICP en 15 centros de la Re- pública Argentina durante los años 2013 al 2016 (Figura 1)9,10. El objetivo primario de ambos registros fue el de conocer la incidencia de muerte de cualquier causa, infarto de miocardio y stroke a un año de seguimiento. En el registro ERACI IV se utilizó un stent de cromocobalto liberador de rapamicina de segunda generación (Firebird 2TM, Microport Corp. Shanghai, China) en pacientes con indicación de revascularización coronaria y enfermedad de dos o tres vasos y/o lesión de tronco coronario izquierdo. En el registro WALTZ se trataron pacientes coronarios, incluidos aquellos con infarto de miocardio con elevación del segmento ST-T (IAMST), y se utilizó un stent de cromocobalto, desnudo (WALTZ ${ }^{\text {ww }}$ Microport Corp, Shanghai, China) con la misma aleación y diseño que el stent Firebird 2. Los criterios de exclusión para ambos registros fueron tener mala función ventricular (fracción de eyección del ventrículo izquierdo< $35 \%$ ), un diámetro de lesión objetivo a tratar $\geq 2,5 \mathrm{~mm}$, angioplastia previa con DES en la arteria culpable, falla renal crónica (clearance de creatinina $>2,5 \mathrm{mg} / \mathrm{dl}$ ), contraindicaciones para el uso de tienopiridinas y mala expectativa de vida. El registro ERACI IV además excluyó a pacientes con IAMST y lesiones de 1 vaso.Para ingresar en ambos registros los pacientes debían firmar el consentimiento informado previamente aprobado por el Ministerio de Justicia de la Nación y ser mayores de 18 años. Ambos protocolos fueron aprobados por los comités de Ética y/o investigación de las instituciones participantes y notificados en la Administración Nacional de Medicamentos, Alimentos y Tecnología Médica (A.N.M.A.T.). El ingreso de los pacientes fue previo al procedimiento basal y la incorporación de datos al registro fue por medio de una plataforma online. El monitoreo de los datos lo realizó el Centro de Estudios en Cardiología Intervencionista (CECI) y en análisis de eventos fue realizado por un comité independiente al estudio. Los pacientes fueron seguidos durante un año mediante entrevistas personales y luego telefónicas a los 3, 6y 12 meses desde el procedimiento basal. 
TABLA 1. Datos demográficos clínicos y angiográficos basales, comparación entre grupos.

\begin{tabular}{|c|c|c|c|c|c|c|}
\hline & $\begin{array}{l}\text { Clopidogrel } \\
(n+/-=240)\end{array}$ & $\begin{array}{c}\text { Prasugrel } \\
(n=80)\end{array}$ & $\begin{array}{c}\text { Ticagrelor } \\
(n=106)\end{array}$ & $\begin{array}{c}\text { Clopidogrel vs } \\
\text { prasugrel (p) }\end{array}$ & $\begin{array}{l}\text { Clopidogrel vs } \\
\text { Ticagrelor }(p)\end{array}$ & $\begin{array}{l}\text { Prasugrel vs } \\
\text { Ticagrelor (p) }\end{array}$ \\
\hline Edad, años & $64,7 \pm 11,9$ & $59,5 \pm 9,6$ & $59,8 \pm 12,0$ & 0,005 & 0.002 & 0.84 \\
\hline Hombres, $\%$ & 78,5 & 89,9 & 85,6 & 0.057 & 0,19 & 0.51 \\
\hline Diabetes,\% & 25,3 & 31,6 & 26,0 & 0.01 & 0,64 & 0.09 \\
\hline HTA,\% & 77,2 & 68,4 & 67,3 & 0.18 & 0.04 & 0.61 \\
\hline $\mathrm{DLP}, \%$ & 68,4 & 65,8 & 64,4 & 0.65 & 0,66 & 0.97 \\
\hline AFEC, $\%$ & 30,4 & 29,1 & 25,0 & 0.66 & 0,43 & 0.31 \\
\hline $\mathrm{TBQ}, \%$ & 40,9 & 53,2 & 36,5 & 0.06 & 0,69 & 0.04 \\
\hline $\mathrm{EC}, \%$ & 43,0 & 39,2 & 32,7 & 0.90 & 0,18 & 0.32 \\
\hline IAM pre, $\%$ & 28,7 & 29,1 & 28,8 & 0.30 & 0,58 & 0.67 \\
\hline Revasc pre, $\%$ & 33,8 & 27,8 & 20,2 & 0.78 & 0,05 & 0.13 \\
\hline CRM previa & 2,9 & 1,3 & 1,9 & 0.92 & 0,76 & 0.87 \\
\hline EVP,\% & 8,0 & 2,5 & 1,9 & 0.15 & 0,06 & 0.85 \\
\hline IRC \% & 2,1 & 1,3 & 1,0 & 0.58 & 0,79 & 1.00 \\
\hline EPOC, \% & 2,5 & 1,3 & 4,8 & 0.46 & 0,40 & 0.29 \\
\hline SS privado $\%$ & 54,4 & 74,7 & 55,8 & 0.006 & 0.64 & 0.006 \\
\hline DAPT pre,\% & 32,9 & 19,0 & 26,9 & 0.06 & 0,25 & 0.90 \\
\hline SCA\% & 57,4 & 67,1 & 76,9 & 0.12 & 0.001 & 0.13 \\
\hline - SCACEST\% & 9,7 & 5,1 & 15,4 & 0,18 & 0,04 & 0,09 \\
\hline
\end{tabular}

HTA: hipertensión arterial; DLP, dislipemia; AFEC: Antecedentes heredofamiliares de enfermedad coronaria; TBQ: Tabaquismo; EC: Enfermedad coronaria conocida; IAM pre: Infarto de miocardio previo; Revasc pre: revascularización previa; CRM: cirugía de by-pass previa; EVP: enfermedad vascular periférica; IRC: insuficiencia renal crónica; EPOC: enfermedad pulmonar obstructiva crónica; SS: sistema de salud privado; DAPT: doble antiagregación plaquetaria previamente; SCA: síndrome coronario agudo; SCACEST: SCA con elevación del segmento ST-T.

TABLA 2. Elección de tienopiridinas de acuerdo al stent utilizado y la complejidad anatómica.

\begin{tabular}{|c|c|c|c|c|c|c|}
\hline & $\begin{array}{l}\text { Clopidogrel } \\
\qquad(n=240)\end{array}$ & $\begin{array}{l}\text { Prasugrel } \\
(n=80)\end{array}$ & $\begin{array}{c}\text { Ticagrelor } \\
(n=106)\end{array}$ & $\begin{array}{l}\text { Clopidogrel vs } \\
\text { prasugrel (p) }\end{array}$ & $\begin{array}{l}\text { Clopidogrel vs } \\
\text { Ticagrelor (p) }\end{array}$ & $\begin{array}{l}\text { Prasugrel vs } \\
\text { Ticagrelor (p) }\end{array}$ \\
\hline Score SYNTAX & $16,7 \pm 13,1$ & $22,4 \pm 12,1$ & $14,5 \pm 11,1$ & $<0,001$ & 0,25 & $<0,001$ \\
\hline Score ERACI & $12,3 \pm 11,5$ & $18,0 \pm 12,6$ & $10,6 \pm 9,4$ & $<0,001$ & 0,38 & $<0,001$ \\
\hline $\mathrm{TCl}$ o DA prox, $\%$ & 35,4 & 45,6 & 26,9 & 0,30 & 0,27 & 0,06 \\
\hline $\mathrm{N}^{\circ}$ stents por pte & $1,76 \pm 0,9$ & $1,62 \pm 0,8$ & $1,62 \pm 0,9$ & 0,20 & 0,96 & 0,34 \\
\hline $\mathrm{N}^{\circ}$ lesiones por pte & $1,5 \pm 0,8$ & $1,5 \pm 0.7$ & $1,4 \pm 0,6$ & 0,20 & 0,18 & 0,19 \\
\hline DES, $\%$ & 57,8 & 62,4 & 32,1 & 0,44 & $<0,001$ & $<0,001$ \\
\hline
\end{tabular}

TCI O DA prox: Enfermedad de tronco coronario izquierdo o arteria descendente anterior proximal. Pte: paciente; DES: stent liberador de fármacos.

TABLA 3. Comparación de eventos clínicos durante la hospitalización y el seguimiento de acuerdo a la tienopiridina utilizada.

\begin{tabular}{|c|c|c|c|c|c|c|}
\hline & $\begin{array}{l}\text { Clopidogrel } \\
(n=240)\end{array}$ & $\begin{array}{c}\text { Prasugrel } \\
(n=80)\end{array}$ & $\begin{array}{l}\text { Ticagrelor } \\
(\mathrm{n}=106)\end{array}$ & $\begin{array}{l}\text { Clopidogrel vs. } \\
\text { prasugrel }(p)\end{array}$ & $\begin{array}{l}\text { Clopidogrel vs. } \\
\text { ticagrelor }(p)\end{array}$ & $\begin{array}{l}\text { Prasugrel vs } \\
\text { Ticagrelor }(p)\end{array}$ \\
\hline Muerte de cualquier causa & $2,9 \%$ & $0,0 \%$ & $4,0 \%$ & 0,24 & 0,62 & 0,06 \\
\hline IAM no fatal & $1,3 \%$ & $2,3 \%$ & $1,0 \%$ & 0,85 & 1,00 & 0,88 \\
\hline ACV & $0,8 \%$ & $0,0 \%$ & $0,0 \%$ & 0,96 & 0,88 & 1,00 \\
\hline $\begin{array}{l}\text { Muerte de cualquier causa, IAM no fa- } \\
\text { tal o ACV }\end{array}$ & $4,2 \%$ & $2,3 \%$ & $5,0 \%$ & 0,43 & 0,75 & 0,34 \\
\hline Revascularización no planeada & $5,9 \%$ & $8,1 \%$ & $9,9 \%$ & 0,46 & 0,74 & 0,53 \\
\hline MACCE & $9,2 \%$ & $9,3 \%$ & $11,9 \%$ & 0,97 & 0,45 & 0,57 \\
\hline Stent trombosis & $0,0 \%$ & $0,0 \%$ & $2,0 \%$ & 1,00 & 0,16 & 0,54 \\
\hline Sangrado mayor & $0,4 \%$ & $0,0 \%$ & $1,0 \%$ & 1,00 & 1,00 & 1,00 \\
\hline
\end{tabular}

IAM: infarto agudo de miocardio; ACV: accidente cerebrovascular; MACCE: muerte de cualquier causa, IAM no fatal, ACV o revascularización no planeada.

\section{Objetivos del registro}

El objetivo primario post hoc del registro ERACI-WALTZ fue el de conocer que tienopiridinas se eligieron al momento de la ICP y su repercusión clínica durante el seguimiento. Los objetivos secundarios fueron muerte de cualquier causa, muerte cardiovascular, infarto de miocardio y accidente cerebrovascular. Además se evalúo la incidencia de eventos duros combinados y de nueva revascularización y se registraron las incidencias de stent trombosis, de sangrado y de cambio o suspensión de medicación antiagregante; y se analizó la elección de drogas inhibidoras de P2Y12 de acuerdo al seguro social de los pacientes incluidos (tomándose como privados aquellos que tenían cobertura médica privada u obras sociales) al momento de la ICP, y si el motivo del procedimiento (SCA o ACE) incidió al momento de la elección del antiagregante plaquetario.

\section{Definición y adjudicación de eventos}

Los eventos se identificaron durante la internación y luego con las visitas de seguimiento. Un comité de eventos adjudicó los mismos de acuerdo a lo recopilado mediante los formularios de reportes de casos electrónicos (CRF) y las historias clínicas, que fueron monitorizadas de forma aleatoria en un $20 \%$ para evaluar eventuales inconsistencias. Al ser este un subanálisis de los estudios ERACI IV y WALTZ, el comité de adjudicación de eventos fue ciego al momento del análisis de acuerdo a elección de doble antiagregación. Los CRF de seguimiento incluyeron preguntas relacionadas a la ocurrencia de eventos isquémicos o de sangrado, hospitalizaciones e intervenciones, programadas o no. Además se preguntó acerca de la continuidad de la DAPT y, en caso de cambios o suspensiones de la misma, la causa. Cuando fue necesario, el investigador de cada centro fue contactado para clarificar o confirmar información de la base de datos.

La definición de muerte se clasificó como cardiovascular cuando pudo adjudicarse a causas cardíacas como infarto, bajo gasto o arritmia fatal, tromboembolismo pulmonar, accidente cerebrovascular o patologías de la aorta y muerte de causa desconocida. Se siguió la clasificación de la tercera definición universal de infarto agudo de miocardio. La defini- 
ción de stent trombosis (definitiva o probable) fue de acuerdo a la Academic Research Consortium (A.R.C.) y de sangrado mayor de acuerdo al TIMI Bleeding Score ${ }^{11,12}$.

\section{Estrategia de la intervención percutánea coronaria y de doble antiagregación}

La estrategia de ICP se planificó previa al procedimiento y el objetivo fue el de lograr una revascularización funcional completa. Se consideró como completa si no quedaban lesiones $>70 \%$ en algún vaso epicárdico coronario, siguiendo el score ERACI, publicado previamente ${ }^{3}$. En resumen, el score ERACI es una modificación al score SYNTAX original que excluye del análisis las lesiones en vasos con diámetros luminales $<2,0 \mathrm{~mm}$ y a lesiones intermedias, $\mathrm{O}$ sea a aquellas con lesiones entre el 50 al $70 \%$ de diámetro, evaluadas por estimación visual. Las oclusiones totales crónicas que irrigaban segmentos aquinéticos no fueron tratadas.

Siendo ambos estudios registros del mundo real, quedó a criterio del operador la dosis de carga y la elección de la tienopiridina al momento del procedimiento basal, siendo de 300 o $600 \mathrm{mg} \mathrm{la}$ dosis de carga del clopidogrel, $180 \mathrm{mg}$ para el ticagrelor y $60 \mathrm{mg}$ para el prasugrel, para luego continuar con las dosis de mantenimiento convencionales, además de la aspirina ${ }^{8}$. Fue mandatorio por protocolo el uso de tienopiridinas durante un año, al menos, para el grupo de pacientes que recibió stent liberador de fármacos (DES) y por seis meses para el grupo de stents desnudos (BMS), quedando a criterio del médico de cabecera la continuación de la DAPT durante períodos más prolongados.

\section{Análisis estadístico}

Se definieron tres grupos de estudio de acuerdo a la droga utilizada: clopidogrel, prasugrel y ticagrelor. Las características entre grupos se compararon utilizando test de chi cuadrado para las variables categóricas y test de ANOVA para las continuas. Se realizaron curvas de Kaplan Meier para analizar la incidencia de eventos a lo largo del tiempo. Además se discriminó a los grupos de acuerdo a la presentación clínica (IAMST o no), tipo de seguro médico (prepago u obra social) y tipo de stent utilizado (DES o BMS) para reconocer posibles variables confundidoras. Se utilizó el paquete estadístico SPSS V.17.1 (IBM, New York, USA)

\section{RESULTADOS}

\section{Características de los pacientes y elección de tienopiridinas}

Los 426 pacientes incluidos en el registro ERACI-WALTZ fueron seguidos durante un año, sin pérdidas durante el seguimiento.El 63\% de los pacientes presentó un síndrome coronario agudo (SCA) al momento del ingreso, siendo 16\%de ellos un IAMST. El 83\% fueron hombres, 27\% diabéticos y 29\% tenían antecedentes de revascularización. El score de SYNTAX fue del $17,25 \pm 12,7$ y el de ERACI $12,9 \pm 11,5$. El $52,8 \%$ de los pacientes recibió un DES, y se implantaron 1,64 stents por paciente en total, contando tanto BMS como DES. Con respecto a la cobertura médica, $57 \%$ tenían cobertura privada, el resto tenían obras sociales. Del total de pacientes incluidos, $56,3 \%$ fueron dados de alta con clopidogrel, $18,8 \%$ con prasugrel y $24,9 \%$ con ticagrelor; en la Tabla 1 se presentan las variables demográficas, clínicas y angiográficas basales relevantes, comparándolas entre los grupos de drogas inhibidoras de P2Y12. A los 6 meses discontinuaron por protocolo o indicación del médico de cabecera, fue del 10,4\% para el clopidogrel, 12,5\% el prasugrel y $8,5 \%$ el ticagrelor; la incidencia de rotación durante el seguimiento

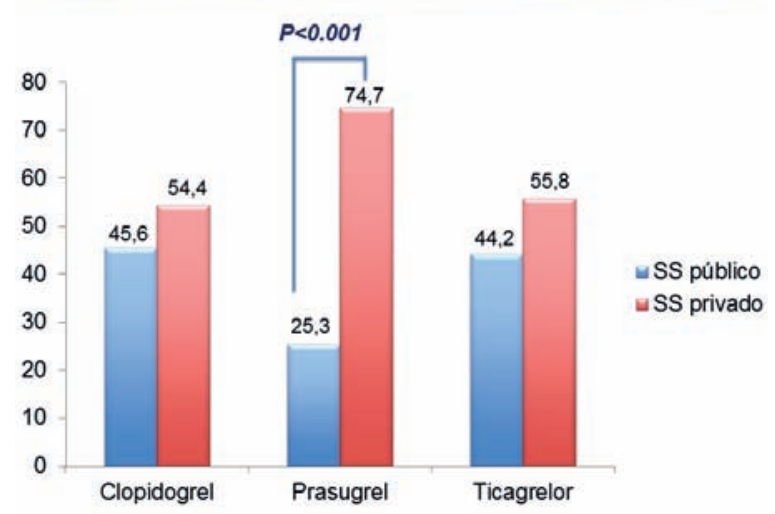

Figura 2. Elección de la tienopiridina y sistema de salud público o privado.

fue de $0,8 \%$ de clopidogrel a ticagrelor, $3,5 \%$ para el prasugrel (se modificó por clopidogrel) y 3,7\% para ticagrelor (a clopidogrel); no hubo rotación de ticagrelor a prasugrel o viceversa. Al evaluar la población de acuerdo al diagnóstico de ingreso, el 57,4\% del grupo clopidogrel sufrió un SCA, 67,1\% del grupo prasugrel (clopidogrel vs. prasugrel, $\mathrm{p}=0.12$ ) y $76,9 \%$ del grupo ticagrelor (clopidogrel $v$ s. ticagrelor, $\mathrm{p}<0,001$ ). Como se ve en la Tabla 1, hubo una preferencia de ticagrelor sobre las otras opciones en los pacientes con IAMEST ( $\mathrm{p}=0,04$ vs. clopidogrel, Tabla $\mathbf{1}$ ).

En cuanto a la elección de la droga y el tipo de stent usado, al $62,4 \%$ de los pacientes del grupo prasugrel se les implantó DES, al $55 \%$ del grupo clopidogrel (clopidogrel $v$ s. prasugrel, $\mathrm{p}=0,44$ ) y solo al $32 \%$ del grupo ticagrelor (clopidogrel $v s$. ticagrelor, $\mathrm{p}$ $<0,001)$;como puede verse en la Tabla 2. Además, el prasugrel se utilizó en el grupo con la anatomía coronaria más compleja, con un score de SYNTAX 22,4 $\pm 16,7$ ( $\mathrm{p}<0,001)$ en comparación contra clopidogrel y ticagrelor, sin presentar diferencias entre clopidogrel y ticagrelor $(\mathrm{p}=0,25)$ (Tabla 2). En todos los casos, se utilizó menos de 2 stents por paciente.

Con respecto al seguro social, solo se vieron diferencias en el grupo prasugrel (25\% de los pacientes del grupo prasugrel fueron de seguro social público y el $75 \%$ del privado, p<0,001) (Figura 2).

\section{Eventos clínicos}

De los eventos duros reportados (muerte de cualquier causa, infarto de miocardio no fatal o accidente cerebrovascular) no hubo diferencias entre las drogas elegidas, tanto combinados como de forma individual, como se describe en Tabla 3. No hubo diferencias en cuanto a la trombosis del stent posible o probable ni en cuanto al sangrado mayor.

Durante el seguimiento de 509£336 días, el análisis de sobrevida libre de muerte de cualquier causa mostró diferencias estadísticamente significativas a favor del prasugrel $(p=0,041)$, resultados que no se replicaron al evaluar MACCE (muerte de cualquier causa, infarto no fatal, accidente cerebrovascular o revascularización no esperada), como se ve en Figura 3, paneles superior e inferior respectivamente.

\section{DISCUSIÓN}

Nuestro estudio muestra que el clopidogrel sigue siendo la primera elección en pacientes a los que se le va a implantar un stent en Argentina, sin importar el cuadro clínico de ingreso, el seguro social o el stent implantado. En uno de cada dos pacientes de este registro multicéntrico se utilizó clopidogrel, 

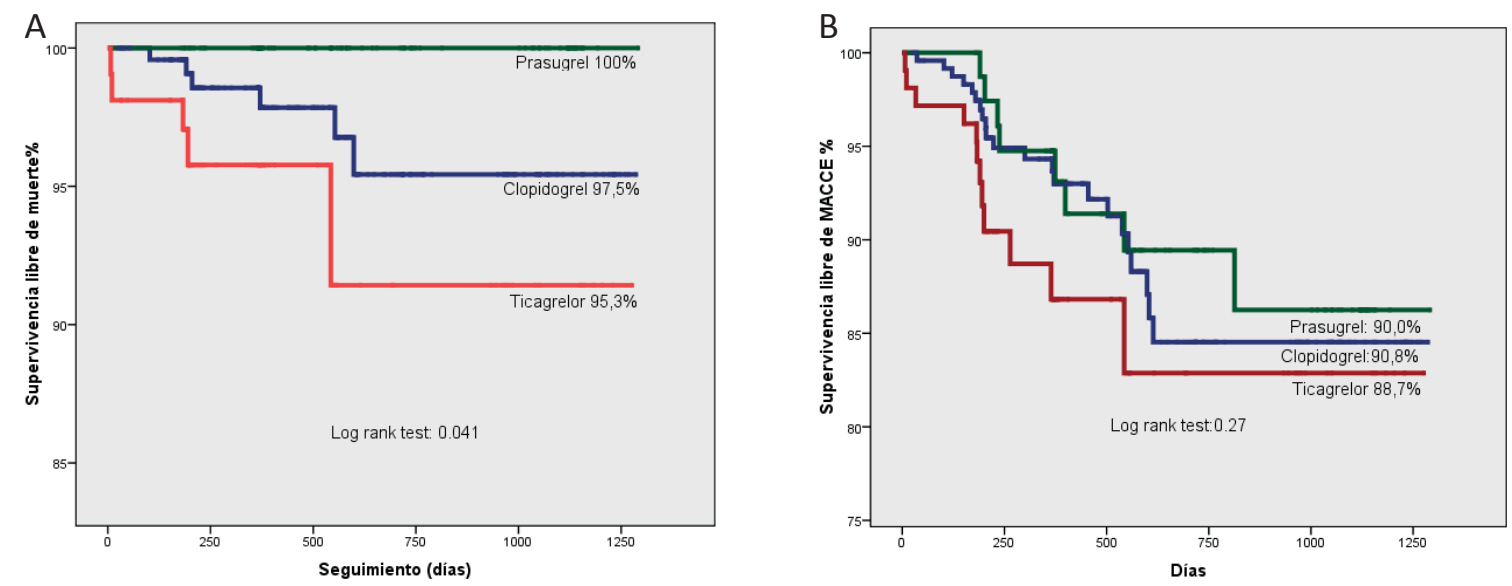

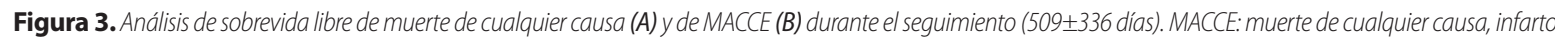
no fatal, accidente cerebrovascular o revascularización no esperada. Figura 2. Elección de la tienopiridina y sistema de salud público o privado.

y la elección de las nuevas drogas inhibidoras de P2Y12 solo aumentó cuando los pacientes tuvieron un síndrome coronario agudo, siendo la primera preferencia el ticagrelor (clopidogrel $57 \%$, prasugrel $67.1 \%$ y ticagrelor $76,9 \%$ ). Esta diferencia porcentual no se ve reflejada en los números finales (Tabla 1).

Teniendo en cuenta que este es un subanálisis para el cual el registro original no fue diseñado, existen limitaciones a la hora de interpretar los datos, la más importante de las cuales es que este no es un ensayo clínico randomizado. Sin embargo, el análisis de las características basales mostró que esta población fue de alto riesgo, con un alto porcentaje de diabéticos, con SYNTAX intermedio o IAMST; sin embargo, la incidencia de eventos duros no fue elevada, adjudicable tal vez a la estrategia de revascularización utilizada, que siguió los lineamientos del ERACI score, donde se recomendó no angioplastiar lesiones con diámetros de referencia pequeños o lesiones intermedias, siguiendo las tendencias actuales de lograr una revascularización funcional completa ${ }^{3}$.

El patrón de uso de clopidogrel del registro puede estar influenciado por los costos de las drogas más que por la evidencia clínica que convalida su elección, ya que existe evidencia de superioridad del ticagrelor y prasugrel sobre el clopidogrel $^{1,2,12}$ y las guías de tratamiento así lo indican ${ }^{13}$. Sin embargo, no hubo diferencias en cuanto a la elección de la DAPT y el seguro social, tanto público como privado. Además, se evaluaron los pacientes desde el ingreso, o sea que se tuvo en cuenta la droga elegida en el ámbito de la internación, momento en el cual no hay gran influencia del costo del DAPT utilizado; y siguiendo este razonamiento, tampoco hubo cambios significativos de las mismas durante el seguimiento, situación que sí puede relacionarse con un problema económico.

El hallazgo de significativa menor mortalidad $(\mathrm{p}=0,04)$ presente en aquellos pacientes que tomaban prasugrel en relación al ticagrelor debe tomarse con cautela dado las diferencias basales pre- sentes tanto clínicas y angiográficas de los pacientes, aquellos tratados con ticagrelor tenían numéricamente mayor incidencia de SCA incluyendo IAMCEST ( $\mathrm{p}=0,12$ y 0,09, respectivamente; Tabla 1) mientras que los tratados con prasugrel tenían mayor score de SYNTAX y ERACI ( $\mathrm{p}<0,001$; Tabla 2) así como mayor número de lesiones de tronco de coronaria izquierda o proximal de arteria descendente anterior ( $p=0,06$; Tabla 2).

Aun con estas limitaciones, estos resultados coinciden con los hallazgos de múltiples registros prospectivos y metaanálisis $^{12,14}$, y recientemente el estudio randomizado ISAR-REACT 5 mostró una reducción significativa de eventos combinados "duros" en el grupo tratado con prasugrel comparado con el grupo ticagrelor $(\mathrm{p}=0,006)$ en pacientes con SCA, población que fue del $66 \%$ en nuestro registro y que podría explicar parte de los resultados ${ }^{15}$.

Si bien el número de pacientes no es grande y esto constituye una limitación de nuestro estudio, los registros previos argentinos en poblaciones más grandes no poseen seguimiento a largo plazo, por lo que los resultados del registro ERACI-WALTZ muestran las tendencias del mundo real en nuestro país, sobre todo a largo plazo ${ }^{16}$.

Por último, existieron otras limitaciones: Se trata de un registro y no de un estudio aleatorizado; además, no fue diseñado específicamente para evaluar la doble antiagregación plaquetaria, en ambos casos se evaluaron stents y ambos estudios se pensaron en base a los mismos y no dirigidos a evaluar el comportamiento de las drogas en los pacientes.

\section{CONCLUSIÓN}

En Argentina los patrones de uso de drogas inhibidoras de P2Y12 no siguen las recomendaciones actuales de las guías de tratamiento. A pesar de la evidencia contemporánea, se utiliza clopidogrel como primera elección, sin importar el cuadro clínico de ingreso, el stent implantado o el tipo de seguro social de los pacientes. 


\section{BIBLIOGRAFÍA}

1. Wiviott SD, Braunwald E, MCCabe CH, et al. TRITON-TIMI 38 Investigators. Prasugrel vs. clopidogrel in patients with acute coronary syndromes. N Engl J Med 2007;357:2001-2015.

2. Wallentin L, Becker RC, Budaj A, et al. Ticagrelor vs. clopidogrel in patients with acute coronary syndromes. N Engl J Med 2009;361:1045-1057.

3. Rodriquez AE, Fernandez-Pereira C, Mieres J, et al. Lowering risk score profile during $P C l$ in multiple vessel disease is associated with low adverse events: The ERACI risk score Cardiovascular Revascularization Medicine 19 (2018) 792-794 793

4. Feres F, Costa RA, Abizaid A, et al. OPTIMIZE Trial Investigators. Three vs twelve months of dual antiplatelet therapy after zotarolimus-eluting stents: the OPTIMIZE randomized trial. JAMA. 2013 Dec 18;310(23):2510-22.

5. Mauri L, Kereiakes DJ, Yeh RW, et al. DAPT Study Investigators.. Twelve or 30 months of dual antiplatelet therapy after drug-eluting stents. NEngl J Med. 2014 Dec 4;371(23):2155-66.

6. Dual antiplatelet therapy after drug-eluting stent implantation: long-term, short-term, tailored or related to stent type? David Antoniucci, Alfredo ERodríguez. Revista Argentina de Cardioangiología 2016;(03):0126-0128

7. Valgimigli M, Campo G,Monti M, et al. Prolonging Dual Antiplatelet Treatment After Grading Stent-Induced Intimal Hyperplasia Study (PRODIGY) Investigators. Short-versus long-term duration of dual-antiplatelet therapy after coronary stenting: a randomized multicenter trial. Circulation. 2012 Apr 24;125(16):2015-26.

8. Valgimigli M, Bueno H, Byrne RA, et al. 2017 ESC focused update on dual antiplatelet therapy in coronary artery disease developed in collaboration with EACTS: The Task Force for dual antiplatelet therapy in coronary artery disease of the European Society of Cardiology (ESC) and of the European Association for Cardio-Thoracic Surgery (EACTS). Eur Heart J. 2018 Jan 14;39(3):213-260.

9. Rodriguez AE, Santaera O, Larribau M, et al. Second vs. first-generation drug-eluting stents in complex lesions subsets: 3 years' follow-up of ERACI IV study. Minerva Cardioangiol. 2017 Feb;65(1):81-90

10. Rodriguez AE, Larribau M, Fernandez-Pereira C et al. One-Year Follow-Up Results From the Observational, Multicenter, Prospective, and Controlled Registry: The WALTZAll-Comers Study. Clin Med Insights Cardiol. 2019 Jun 27:13:1179546819854059.
11. Mehran R, RaoSV, BhattDL, Gibson CM, et al. Standardized bleeding definitions for cardiovascular clinical trials: a consensus reportfrom the bleeding academic research consortium. Circulation. 123 (23): 2736-47.

12. Rodríguez AE, Rodriguez-Granillo AM, Ascarrunz SD, Peralta-Bazan $F$ and Cho MY. Did Prasugrel and Ticagrelor Offer the Same Benefit in Patients with Acute Coronary Syndromes after Percutaneous Coronary Interventions Compared to Clopidogrel? Insights from Randomized Clinical Trials, Registries and Meta-analysis. Curr Pharm Des. 2018;24(4):465-477.

13. Levine GN, Bates ER, Bittl JA, et al. 2016 ACC/AHA Guideline Focused Update on Duration of Dual Antiplatelet Therapy in Patients With Coronary Artery Disease: A Report of the American College of Cardiology/ American Heart Association Task Force on Clinical Practice Guidelines: An Update of the 2011 ACCF/AHA/SCAl Guideline for Percutaneous Coronary Intervention, 2011 ACCF/AHA Guideline for Coronary Artery Bypass Graft Surgery, 2012 ACC/AHA/ACP/AATS/PCNA/SCAI/STS Guideline for the Diagnosis and Management of Patients With Stable Ischemic Heart Disease, 2013 ACCF/AHA Guideline for the Management ofST-Elevation Myocardial Infarction, 2014 AHA/ACC Guideline for the Management of Patients With Non-ST-Elevation Acute Coronary Syndromes, and 2014 ACC/AHA Guideline on Perioperative Cardiovascular Evaluation and Management of Patients Undergoing Noncardiac Surgery. Circulation. 2016 Sep 6;134(10):e123-55

14. Olier I, Sirker A, Hildick-Smith DJR, et al. British Cardiovascular Intervention Society and the National Institute for Cardiovascular Outcomes Research.Association of different antiplatelet therapies with mortality after primary percutaneous coronary intervention. Heart. 2018 Oct; 104(20):1683-1690.

15. SchüpkeS, Neumann FJ, Menichelli M, et al. Ticagrelor or Prasugrel in Patients with Acute Coronary Syndromes. N Engl J Med. 2019 Sep 1.

16. Fernández-Pereira C, Descalzo A, Cherro A, et al. Resultados intrahospitalarios en pacientes con infarto agudo de miocardio tratados con angioplastia dentro del Registro Argentino de Angioplastia Coronaria ( $R a-$ dAC). Revista Argentina de Cardioangiología Intervencionista 2012;(01): 0028-0036. 